\title{
Etude des paramètres sériques biochimiques : le cas des lapins (neozelandais - cunistar) de Côte d'ivoire.
}

\author{
Founzégué A. Coulibaly ${ }^{1 *}$, Adama Coulibaly ${ }^{1}$, Jean D. N'Guéssan ${ }^{1}$, Koffi G. Kouamé $^{2}$, Allico J. DJaman $^{1,3} \&$ Frédéric $^{\prime}$ \\ GUÉDÉ-GUINA'. \\ ${ }^{1}$ Laboratoire de Pharmacodynamie-Biochimique. \\ ${ }^{2}$ Laboratoire de Nutrition - Pharmacologie. U.F.R. Biosciences Université de Cocody Abidjan - Côte d'Ivoire. \\ ${ }^{3}$ Laboratoire de Biochimie clinique et fondamentale-Institut Pasteur de Côte d'Ivoire. \\ *Auteur pour les Correspondances (E-mail : founzegue@yahoo.fr) \\ Reçu le 30-06-2005, accepté le 08-01-2007.
}

\section{Résumé}

L'étude des paramètres sériques biochimiques chez les lapins (Néozélandais - cunistar) en Côte d'Ivoire répond à une insuffisance des bases données. Les valeurs obtenues sur l'ensemble des sérums des animaux ont permis d'observer les moyennes au niveau de la glycémie $(0,79 \pm 0,18 \mathrm{~g} / \mathrm{l})$ et des métabolites tels que, l'urée $(0,42 \pm 0,10 \mathrm{~g} /$ I), la créatinine $(6,88 \pm 1,66 \mathrm{mg} / \mathrm{l})$, l'acide urique $(5,423,75 \mathrm{mg} / \mathrm{l})$, le cholestérol total $(0,55 \pm 0,13 \mathrm{mg} / \mathrm{l})$, les triglycérides $(2,18 \pm 1,23 \mathrm{~g} / \mathrm{l})$, les protéines totales $(26,00 \pm 15,16 \mathrm{~g} / \mathrm{l})$, les bilirubines totales $(7,25 \pm 1,07 \mathrm{mg} / \mathrm{l})$ et les bilirubines directes $1,58 \pm 0,38 \mathrm{mg} / \mathrm{l}$. Concernant les enzymes, les déterminations des valeurs ont été faites pour la transaminase alanine - aminotransférase $(45,52 \pm 20,54 \mathrm{UI} / \mathrm{l})$, la transaminase aspartate - aminotransférase $(21,24 \pm 9,89 \mathrm{UI} / \mathrm{l})$, les phosphatases alcalines $(432,66 \pm 207,8 \mathrm{UI} / \mathrm{l})$, la ã Glutamyl transférase $(24,24 \pm 15,21 \mathrm{UI} / \mathrm{l})$, les créatine phosphokinases (954 $\pm 343,4 \mathrm{UI} / \mathrm{l})$, les lactates déshydrogénases (1135 $\pm 335,93 \mathrm{UI} / \mathrm{l})$ et enfin les amylases (114,72 $\pm 27,99 \mathrm{UI} / \mathrm{l})$. Par ailleurs, les moyennes des ions ont été déterminées pour le calcium ( $94 \pm 4,43 \mathrm{mg} / \mathrm{l})$, le magnésium (15,72 $\pm 2,49 \mathrm{mg} /$ I), le phosphore $(26,70 \pm 10,51 \mathrm{mg} / \mathrm{l})$, le fer sérique $(1,33 \pm 0,74 \mathrm{mg} / \mathrm{l})$, le sodium $(141,89 \pm 3,96 \mathrm{mg} / \mathrm{l})$, le potassium $(3,89 \pm 0,38 \mathrm{mg} / \mathrm{l})$ et enfin le chlore $(100,85 \pm 3,04 \mathrm{mg} / \mathrm{l})$.

En conclusion, les valeurs obtenues en zone tropicale nécessitent une évaluation avec un échantillonnage plus grand pour des comparaisons avec des données européennes.

Mots clés : paramètr.es sériques, Néozélandais -Cunistar, lapin.

\section{Abstract}

Determination of serum biochimical parameters : The case of rabbits (neozelandais-cunistar) of Cote d'Ivoire.

Study of biochemical serum constituents among rabbits (Néozélandais-cunistar) in Côte d'Ivoire is carried out as a result insufficiency of available data. Values acquired from all animals serum allowed to look at glycemia $(0.79 \pm$ $0.18 \mathrm{~g} / \mathrm{l})$ and metabolites related averages such as, urea $(0.42 \pm 0.10 \mathrm{~g} / \mathrm{l})$, creatinine $(6.88 \pm 1.66 \mathrm{mg} / \mathrm{l})$, uric acid (5.42 $\pm 3.75 \mathrm{mg} / \mathrm{l})$, total cholesterol $(0.55 \pm 0.13 \mathrm{mg} / \mathrm{l})$, triglycol $(2.18 \pm 1.23 \mathrm{~g} / \mathrm{l})$, total proteins $(26.00 \pm 15.16 \mathrm{~g} / \mathrm{l})$, total bilirubins (7.25 $\pm 1.07 \mathrm{mg} / \mathrm{l})$ and bilirubins direct $(1.58 \pm 0.38 \mathrm{mg} / \mathrm{l})$. Concerning enzymes, averages were worked out for alanine - aminotransferase (45.52 $\pm 20.54 \mathrm{UI} / \mathrm{I})$, aspartate - aminotransferase (21.24 $\pm 9.89 \mathrm{UI} / \mathrm{I})$, phosphatases alkaline (432.66 $\pm 207.8 \mathrm{UI} / \mathrm{I})$, ã Glutamyl transférase (24.24 $\pm 15.21 \mathrm{UI} / \mathrm{I})$, créatine kinase (954 $\pm 343.4 \mathrm{UI} / \mathrm{l})$, lactate deshydrogenase (1135 $\pm 335.93 \mathrm{UI} / \mathrm{l})$ and the amylases (114.72 $\pm 27.99 \mathrm{UI} / \mathrm{l})$. Besides as to ions, the averages were determined for calcium (94 $\pm 4.43 \mathrm{mg} / \mathrm{l})$, magnesium (15.72 $\pm 2.49 \mathrm{mg} / \mathrm{l})$, phosphor (26.70 $\pm 10.51 \mathrm{mg} / \mathrm{l})$, iron serum $(1.33 \pm 0.74 \mathrm{mg} / \mathrm{l})$, sodium (141.89 $\pm 3.96 \mathrm{mg} / \mathrm{l})$, potassium (3.89 $\pm 0.38 \mathrm{mg} / \mathrm{l})$ and chlorine $(100.85 \pm 3.04 \mathrm{mg} / \mathrm{l})$. In short, the values obtained in tropical area require assessment with a larger sampling for comparisons with European data.

Keywords: serum parameters, Néozélandais - Cunistar, rabbit. 


\section{Introduction}

L'élevage des lapins occupe une place importante actuellement dans les pays en développement comme la Côte d'Ivoire. Les espèces élevées sont généralement utilisées comme sources de protéines animales pour l'alimentation humaine où comme modèles biologiques pour les expériences in vivo de laboratoire; Ceci exige une bonne hygiène corporelle et une excellente santé clinique dans un environnement tropical sujet à de nombreuses pathologies infectieuses.

Des études préliminaires d'Adjanahoun et Aké (1979) avaient recensé environ 5000 plantes ayant des vertus médicinales. Afin de préciser les modes d'action et / où leurs effets secondaires pour contribuer au développement de la pharmacopée africaine, il est entrepris une étude de biotolérance de certaines plantes médicinales couramment utilisées dans la pharmacopée africaine.

Ces études par rapport aux références de Sibernagl et Despapoulos (1992) et ceux de Claude (1988), nécessitent une approche dynamique pour préciser le site où les cibles des substances actives agissent, mais aussi l'action de leurs effets pharmacologiques sur des paramètres sériques biochimiques et / ou physiologiques. En 2003, une étude de Coulibaly a permis d'enregistrer $10,3 \%$ des travaux réalisés qui ont pris comme modèle biologique les lapins par rapport aux 9,2\% des travaux réalisés dans les laboratoires d'Europe sur les lapins selon Laroche et Rousselet (1990).

Parmi les travaux effectués en Côte d'Ivoire, Coulibaly et al. (2003), Konin (2002), Kadja (1999) et Djédjé (2002) ont pris comme références des paramètres sériques des valeurs établies soit en dehors des conditions tropicales (Lebas, 2002 ; Boucher \& Mouaille, 1996), soit avec des données relatives à l'homme (Robert, 1980 ; Konard \& Cornélius, 1973 ; Eastham, 1978).

Face à cette problématique de la disponibilité des paramètres biologiques standards des lapins en zone tropicale, ce travail a pour objectif d'étudier les valeurs des paramètres sériques biochimiques chez les lapins (NéozélandaisCunistar) en Côte d'Ivoire afin d'établir une similitude entre celles observées dans ce pays et les valeurs observées en dehors des conditions tropicales.

\section{Matériels et Méthodes}

\subsection{Matériels}

\subsubsection{Matériel biologique}

Les lapins élevés dans la plupart des fermes de la localité de Bingerville (Côte d'Ivoire) sont issus du croisement des races Néozélandaise et Cunistar. Pour cette étude, trente (30) lapins (15 mâles et 15 femelles) âgés de deux (2) mois ont été sélectionnés.

\subsubsection{Matériel de laboratoire}

La réalisation du dosage des différents paramètres a nécessité l'utilisation de matériel composé d'une centrifugeuse JOUAN, d'un spectrophotomètre de flamme SEAC $f p 20$, d'un spectrophotomètre UV visible HITACHI 704, d'un ensemble de kits de dosage de référence (BIOMERIEUX, BIOSYSTEME et BIOLABO).

\subsection{Méthodes}

\subsubsection{Traitement des lapins}

Les animaux utilisés dans cette étude ont un poids corporel d'environ un (1) kilogramme. Ils sont élevés en lots de trois (3) lapins (mâles ou femelles) par cage, acclimatés pendant deux (2) semaines et alimentés quotidiennement avec $150 \mathrm{~g}$ de granulés utilisés comme nutriment d'entretien en provenance d'IVOGRAINS.

\subsubsection{Conditionnement des prélèvements}

Des prélèvements sanguins uniques au niveau de la veine marginale de l'oreille sont effectués dans la matinée, entre six (6) heures et huit (8) heures, sur chaque animal soumis à jeun pendant environ dix (10) heures. Ces prélèvements sont centrifugés à 3000 tours par minute pendant dix (10) minutes. Les sérums obtenus après décantation sont ensuite congelés à $-20^{\circ} \mathrm{C}$ pour la détermination des valeurs des différents paramètres biochimiques. 


\subsubsection{Paramètres sériques analysés}

A l'exception du sodium et du potassium qui sont analysés par un spectrophotomètre de flamme SEAC $f p$ 20, les autres paramètres biochimiques sont analysés à l'aide d'un spectrophotomètre
UV visible HITACHI 704 avec différentes longueurs d'onde selon les méthodes de références indiquées par les kits utilisés. Les méthodes des paramètres analysés dans cette étude sont indiquées dans les tableaux 1, 2 et 3 .

Tableau 1 : Méthodes d'analyses des métabolites

\begin{tabular}{llc}
\hline Paramètres & Méthodes colorimétriques & $\lambda(\mathrm{nm})$ \\
\hline Glycémie & glucose oxydase et peroxydase & 500 \\
Urée & uréase & 600 \\
Créatinine & cinétique en milieu alcalin et avec acide picrique & 500 \\
Acide urique & uricase et de peroxydase. & 510 \\
Protéines totales & ions cuivriques et en milieu alcalin & 550 \\
Cholestérol & cholestérol estérase, d'oxydase et de peroxydase & 500 \\
Triglycérides & lipase, glycérol kinase, oxydase et peroxydase & 500 \\
Bilirubines & - Directes : acide sulfanilique & 550 \\
\hline
\end{tabular}

Tableau 2 : Méthodes d'analyses des enzymes

\begin{tabular}{lll}
\hline Paramètres & Méthodes cinétiques enzymatiques & $\lambda(\mathrm{nm})$ \\
\hline GPT & disparition du NADH & 340 \\
\hline GOT & disparition du NADH & 340 \\
\hline PAL & disparition de PNP & 405 \\
\hline LDH & disparition de NADH + H+ & 340 \\
\hline CPK & disparition de NADH + H+ & 340 \\
\hline Y GT & vitesse de formation de la phospho nitroaniline & 405 \\
\hline$\alpha$ amylase & \multirow{2}{*}{ formation de la 2 chloro 4 nitrophénol } & 405 \\
\hline
\end{tabular}

Tableau 3 : Méthodes d'analyses des ions

\begin{tabular}{llc}
\hline Paramètres & Méthodes colorimétriques & $\lambda(\mathrm{nm})$ \\
\hline Chlore & en milieu acide nitrique et en présence d'ions thiocyanate & 500 \\
Calcium & en alcalin et en présence de l'O-crésol phtaléine. & 500 \\
Magnésium & en alcalin et en présence de calmagite & 530 \\
Fer sérique & en milieu acide et en présence de FERENE & 600 \\
Phosphore & en milieu acide et en présence de molybdate d'ammonium & 600 \\
Paramètres & Méthodes de spectrométrie de flamme & $\lambda$ \\
sodium & à température de 2000 Kelvin & 589 \\
Potassium & à température de 2000 ${ }^{\circ}$ Kelvin & 767 \\
\hline
\end{tabular}

\section{.2.2.4. Analyses statistiques}

Les analyses biologiques pour chacun des paramètres sur les animaux ont permis de récapituler les données statistiques selon les méthodes décrites par Dagnelie (1975), Bouyer
(1997) et Bouyer et al. (1997):

- La valeur moyenne : moy

- La valeur maximale : maxi

- La valeur minimale : mini

- La valeur de l'écart type : s 


\section{Résultats}

Pour chaque paramètre biochimique étudié, les valeurs obtenues avec l'ensemble des lapins élevés en zone tropicale ont permis de présenter des résultats qui sont mentionnés dans les tableaux 4, 5, 6. Dans ces tableaux se trouvent aussi les valeurs observées chez les lapins en Europe (en dehors des zones tropicales) selon Lebas (2002) pour les enzymes d'une part et d'autre part selon Boucher et Mouaille (1996) pour les métabolites et les ions.

Tableau 4 : Récapitulatif des valeurs sériques des métabolites chez les lapins

\begin{tabular}{|c|c|c|c|c|c|c|c|}
\hline & & \multirow{2}{*}{\multicolumn{6}{|c|}{ Valeurs }} \\
\hline & & & & & & & \\
\hline \multirow{2}{*}{ Paramètres } & \multirow{2}{*}{ Unité } & \multicolumn{4}{|c|}{ Tropicales } & \multicolumn{2}{|c|}{ Européennes } \\
\hline & & moy & $\max$ & $\min$ & $\sigma$ & $\max$ & $\min$ \\
\hline Glycémie & $g / l$ & 0,79 & 1,12 & 0,48 & 0,18 & 1,50 & 0,75 \\
\hline Urée & $g / 1$ & 0,42 & 0,78 & 0,29 & 0,10 & 0,23 & 0,17 \\
\hline Créatinine & $\mathrm{mg} / \mathrm{l}$ & 6,88 & 11,3 & 4,3 & 1,66 & 18 & 8 \\
\hline Acide urique & $\mathrm{mg} / \mathrm{l}$ & 5,42 & 17 & 2 & 3,75 & 1 & I \\
\hline Cholestérol total & $g / l$ & 0,55 & 0,87 & 0,36 & 0,13 & 0,80 & 0,10 \\
\hline Triglycérides & $g / 1$ & 2,18 & 4,85 & 0,46 & 1,23 & 1,56 & 1,24 \\
\hline Protéines totales & $\mathrm{g} / \mathrm{I}$ & 26,00 & 54,4 & 11,2 & 15,16 & 85 & 54 \\
\hline Bilirubines totales & $\mathrm{mg} / \mathrm{l}$ & 7,25 & 9,1 & 4,7 & 1,07 & 7,4 & 2,5 \\
\hline Bilirubines directes & $\mathrm{mg} / \mathrm{l}$ & 1,58 & 3 & 1 & 0,38 & l & l \\
\hline
\end{tabular}

Tableau 5 : Récapitulatif des valeurs sériques des enzymes chez les lapins

\begin{tabular}{|c|c|c|c|c|c|c|c|}
\hline \multirow{3}{*}{ Paramètres } & \multirow{3}{*}{ Unité } & \multicolumn{6}{|c|}{ Valeurs } \\
\hline & & \multicolumn{4}{|c|}{ Tropicales } & \multicolumn{2}{|c|}{ Européennes } \\
\hline & & moy & $\max$ & $\min$ & $\sigma$ & $\max$ & $\min$ \\
\hline$\overline{\text { GPT }}$ & $\mathrm{UI} / \mathrm{I}$ & 45,52 & 97 & 17 & 20,54 & 25,5 & 6,5 \\
\hline GOT & UI / I & 21,24 & 46 & 9 & 9,89 & 18,5 & 3,2 \\
\hline PAL & $\mathrm{UI} / \mathrm{I}$ & 432,66 & 679 & 104 & 207,8 & I & 1 \\
\hline y GT & $\mathrm{UI} / \mathrm{I}$ & 24,24 & 67 & 10 & 15,21 & 6,5 & 3,5 \\
\hline CPK & $\mathrm{UI} / \mathrm{I}$ & 954 & 1477 & 198 & 343,4 & i & I \\
\hline $\mathrm{LDH}$ & $\mathrm{UI} / \mathrm{I}$ & 1135 & 1911 & 700 & 335,93 & 257 & 193 \\
\hline Amylases & $\mathrm{UI} / \mathrm{I}$ & 114,72 & 179 & 70 & 27,99 & 1 & 1 \\
\hline
\end{tabular}

Tableau 6 : Récapitulatif des valeurs sériques des ions chez les lapins

\begin{tabular}{lrrrrrrr} 
& & \multicolumn{6}{c}{ Valeurs } \\
\cline { 3 - 8 } Paramètres & \multirow{2}{*}{ Unité } & \multicolumn{5}{c}{ Tropicales } & \multicolumn{4}{c}{ Européennes } \\
\cline { 3 - 8 } & & moy & $\max$ & $\min$ & $\sigma$ & $\max$ & $\min$ \\
\hline Calcium & $\mathrm{mg} / \mathrm{I}$ & 94 & 98 & 78 & 4,43 & 98 & 73 \\
magnésium & $\mathrm{mg} / \mathrm{I}$ & 15,72 & 20 & 12 & 2,49 & 57 & 47 \\
Phosphore & $\mathrm{mg} / \mathrm{I}$ & 26,70 & 51 & 14 & 10,51 & 62 & 40 \\
Fer sérique & $\mathrm{mg} / \mathrm{I}$ & 1,33 & 3,49 & 0,49 & 0,74 & & 2 \\
Sodium & $\mathrm{mEq} / \mathrm{I}$ & 141,89 & 149 & 135 & 3,96 & 150 & 142 \\
Potassium & $\mathrm{mEq} / \mathrm{I}$ & 3,89 & 4,7 & 3,3 & 0,38 & 6 & 5,5 \\
Chlore & $\mathrm{mEq} / \mathrm{I}$ & 100,85 & 107 & 96 & 3,04 & 110 & 100 \\
\hline
\end{tabular}

\section{Discussion}

La présente étude sur les paramètres sériques biochimiques des lapins Néozélandais-cunistar élevés dans les conditions tropicales, répond à une insuffisance de données scientifiques aussi bien pour les expériences aux laboratoires que pour l'évaluation de l'état de santé des lapins en Côte d'Ivoire. Les résultats obtenus intervenant dans le cadre des études de la biotolérance des 
plantes médicinales, constituent une base de données comme celles obtenues par Lebas (2002) et par Boucher et Mouaille (1996). L'échantillonnage utilisé dans cette étude avec un nombre de trente (30) lapins (mâles et femelles) limite dans une certaine mesure les analyses des comparaisons statistiques avec des données des travaux antérieurs réalisés en Europe. Cependant, les valeurs observées avec les paramètres biochimiques en Côte d'Ivoire permettent de faire un constat par rapport aux valeurs obtenues en Europe.

Les Métabolites Les valeurs moyennes observées avec la glycémie de 0,79 $\pm 0,18 \mathrm{~g} / \mathrm{l}$, avec les bilirubines totales de 7,25 $\pm 1,07 \mathrm{mg} / \mathrm{l}$, avec la créatinine de $6,88 \pm 1,66 \mathrm{mg} / \mathrm{l}$ et avec le cholestérol de 0,55 $\pm 0,13 \mathrm{~g} / \mathrm{l}$ sont respectivement compris dans un intervalle de $1,12 \mathrm{~g} / \mathrm{l}$ et $0,48 \mathrm{~g} / \mathrm{l}$, de $9,10 \mathrm{mg} / \mathrm{l}$ et $4,70 \mathrm{mg} / \mathrm{l}$, de $11,30 \mathrm{mg} / \mathrm{l}$ et 4,30 $\mathrm{mg} / \mathrm{l}$ et enfin de $0,87 \mathrm{~g} / \mathrm{l}$ et $0,36 \mathrm{~g} / \mathrm{l}$. Pour ces différents paramètres en zone tropicale, l'intervalle des valeurs obtenues pourrait partiellement se superposer avec les valeurs obtenues par Boucher et Mouaille (1996) qui trouvent que les lapins européens ont une glycémie comprise $1,50 \mathrm{~g} / \mathrm{l}$ et $0,75 \mathrm{~g} / \mathrm{l}$, des bilirubines totales comprises entre $7,4 \mathrm{mg} / \mathrm{l}$ et $2,5 \mathrm{mg} / \mathrm{l}$, une créatinémie comprise entre $18 \mathrm{mg} /$ I et $8 \mathrm{mg} / \mathrm{l}$ et enfin une cholestérolémie comprise entre 0,80 $\mathrm{g} / \mathrm{l}$ et 0,10 $\mathrm{g} / \mathrm{l}$. Par ailleurs, certaines moyennes obtenues avec l'urée de 0,42 $\pm 0,10$ $\mathrm{g} / \mathrm{l}$, avec les triglycérides de 2,18 $\pm 1,23 \mathrm{~g} / \mathrm{l}$ et avec les protéines totales de 26,00 $\pm 15,16 \mathrm{~g} / \mathrm{l}$ sont respectivement comprises entre les intervalles de 0,78 $\mathrm{g} / \mathrm{l}$ et $0,29 \mathrm{~g} / \mathrm{l}$, de $4,85 \mathrm{~g} / \mathrm{l}$ et $0,46 \mathrm{~g} / \mathrm{l}$ et enfin de 54,4 g/l et 11,2 $\mathrm{g} / \mathrm{l}$. Pour ces paramètres, les intervalles des valeurs observées dans les conditions tropicales sont différents de celles obtenues par Boucher et Mouaille (1996) qui ont trouvé que les lapins ont des valeurs de l'urée comprises entre $0,23 \mathrm{~g} / \mathrm{l}$ et $0,17 \mathrm{~g} / \mathrm{l}$, des triglycérides comprises entre 1,56 $\mathrm{g} / \mathrm{l}$ et $1,24 \mathrm{~g} / \mathrm{l}$ et enfin des protéines totales comprises entre $85 \mathrm{~g} / \mathrm{l}$ et $54 \mathrm{~g} / \mathrm{l}$. En outre, l'absence des données sur les paramètres tels que l'acide urique et les bilirubines directes avec les données de Boucher et Mouaille (1996) permet de présenter les valeurs observées chez les lapins élevés en zone tropicale qui sont comprises entre $17 \mathrm{mg} / \mathrm{l}$ et $2 \mathrm{mg} / \mathrm{l}$ avec une moyenne de 5,42 $\pm 3,75 \mathrm{mg} / \mathrm{l}$ pour l'acide urique et entre $3 \mathrm{mg} / \mathrm{l}$ et $1 \mathrm{mg} / \mathrm{l}$ avec une moyenne de $1,58 \pm 0,38 \mathrm{mg} / \mathrm{l}$ pour les bilirubines directes.
Les enzymes_Au regard de l'activité enzymatique sérique, les valeurs moyennes observées avec les transaminases indiquent 45,52 \pm 20,54 UI/I pour GPT et 21,24 \pm 9,89 UI/ I pour GOT. Ces valeurs sont comprises dans un intervalle de $97 \mathrm{UI} / \mathrm{l}$ et $17 \mathrm{UI} / \mathrm{I}$ pour les GPT et de $46 \mathrm{UI} / \mathrm{l}$ et $9 \mathrm{UI} / \mathrm{I}$ pour les GOT. Par rapport aux valeurs obtenues en Europe avec Lebas (2002) qui indiquent que les transaminases sont dans un intervalle compris entre $25,5 \mathrm{UI} / \mathrm{I}$ et $6,5 \mathrm{UI} / \mathrm{I}$ pour les GPT et entre 18,5 et $3,2 \mathrm{UI} / \mathrm{l}$ pour les GOT. Les valeurs de GPT et de GOT obtenues en zone tropicale peuvent être partiellement superposables avec celles obtenues en Europe. Par ailleurs, les valeurs des activités enzymatiques observées indiquent avec g GT une moyenne de 24,24 \pm 15,21 UI/I dans un intervalle compris entre $67 \mathrm{UI} / \mathrm{l}$ et $10 \mathrm{UI} / \mathrm{l}$ alors qu'avec LDH la moyenne est de $1135 \pm 335,93 \mathrm{UI} /$ I dans un intervalle compris entre $1911 \mathrm{UI} / \mathrm{I}$ et 700 UI/I. Ces valeurs observées chez les lapins en zone tropicale ne semblent pas être superposables avec les données européennes de Lebas (2002) qui indiquent pour g GT un intervalle compris entre 6,5 UI/l et 3,5 UI// et pour LDH un intervalle compris entre $257 \mathrm{UI} / \mathrm{l}$ et $193 \mathrm{UI} / \mathrm{l}$.

En l'absence de données des activités enzymatiques comme les PAL, CPK et les amylases avec Lebas (2002), les valeurs obtenues chez les lapins en zone tropicale indiquent une moyenne de 432,66 \pm 207,8 UI/I pour les PAL dans un intervalle compris entre $679 \mathrm{UI} / \mathrm{l}$ et $104 \mathrm{UI} / \mathrm{l}$, une moyenne de $954 \pm 343,4$ UI/I pour les CPK dans un intervalle compris entre $1477 \mathrm{UI} / \mathrm{l}$ et $198 \mathrm{UI} / \mathrm{l}$ et enfin une moyenne de $114,72 \pm 27,99 \mathrm{UI} / \mathrm{l}$ pour les amylases dans un intervalle compris entre $179 \mathrm{UI} / \mathrm{l}$ et $70 \mathrm{UI} / \mathrm{I}$.

Les ions_Les éléments ioniques sériques dosés chez les lapins en zone tropicale donnent des valeurs moyennes de $94 \pm 4,43 \mathrm{mg} / \mathrm{l}$ pour le calcium dans un intervalle compris entre $98 \mathrm{mg} /$ I et $78 \mathrm{mg} / \mathrm{l}$, de $26,70 \pm 10,51 \mathrm{mg} / \mathrm{l}$ pour le phosphore dans un intervalle compris entre 51 $\mathrm{mg} / \mathrm{l}$ et $14 \mathrm{mg} / \mathrm{l}$, de $1,33 \pm 0,74 \mathrm{mg} / \mathrm{l}$ pour le fer sérique dans un intervalle compris entre 3,49 $\mathrm{mg} / \mathrm{l}$ et $0,49 \mathrm{mg} / \mathrm{l}$, de 141,89 $\pm 3,96 \mathrm{mEq} / \mathrm{l}$ pour le sodium dans un intervalle compris entre 149 $\mathrm{mEq} / \mathrm{l}$ et $135 \mathrm{mEq} / \mathrm{l}$ et enfin de 100,85 \pm 3,04 $\mathrm{mEq} / \mathrm{l}$ pour le chlore dans un intervalle compris entre $107 \mathrm{mEq} / \mathrm{l}$ et $96 \mathrm{mEq} / \mathrm{l}$. Ces valeurs observées pourraient être partiellement superposables avec les données de Boucher et 
Mouaille (1996) qui indiquent un intervalle compris entre $98 \mathrm{mg} / \mathrm{l}$ et $73 \mathrm{mg} / \mathrm{l}$ pour le calcium, entre $62 \mathrm{mg} / \mathrm{l}$ et $40 \mathrm{mg} / \mathrm{l}$ pour le phosphore, entre $150 \mathrm{mEq} / \mathrm{l}$ et $142 \mathrm{mEq} / \mathrm{l}$ pour le sodium, entre $110 \mathrm{mEq} / \mathrm{l}$ et $100 \mathrm{mEq} / \mathrm{l}$ pour le chlore et enfin aux alentours de $2 \mathrm{mg} / \mathrm{l}$ pour le fer sérique. Par ailleurs, les valeurs obtenues indiquent pour le magnésium une moyenne de 15,72 $\pm 2,49$ $\mathrm{mg} / \mathrm{l}$ dans un intervalle compris entre $20 \mathrm{mg} / \mathrm{l}$ et $12 \mathrm{mg} / \mathrm{l}$ et pour le potassium une moyenne de $3,89 \pm 0,38 \mathrm{mEq} / \mathrm{l}$ dans un intervalle compris entre $4,7 \mathrm{mEq} / \mathrm{l}$ et $3,3 \mathrm{mEq} / \mathrm{l}$. Ces valeurs observées chez les lapins en zone tropicale ne semblent pas être superposables aux données de Boucher et Mouaille (1996) qui indiquent des valeurs du magnésium comprises entre $57 \mathrm{mg} /$ I et $47 \mathrm{mg} / \mathrm{l}$ et celles du potassium comprises entre $6 \mathrm{mEq} / \mathrm{l}$ et $5,5 \mathrm{mEq} / \mathrm{l}$.

D'une manière générale par rapport aux valeurs européennes, certains paramètres sériques obtenus sous les tropiques chez les lapins comme les enzymes (GOT, GPT, gGT, CPK) présentent des valeurs relativement élevées alors que les ions (magnésium, phosphore et potassium) présentent des valeurs relativement faibles. Par contre, au niveau des métabolites comme les protéines et la créatinine, ces valeurs tropicales sont faibles tandis que l'urée et les triglycérides ont des valeurs élevées. Ce qui permet d'envisager au regard des références de Sibernagl et Despapoulos (1992) des différences physiologiques liées aux facteurs tels que l'alimentation, le climat et les conditions d'élevages.

\section{Conclusion}

En conclusion, les valeurs des différents paramètres sériques biochimiques composés des métabolites, des enzymes et des ions obtenus dans la présente étude malgré les limites expérimentales, ont été utilisées comme données de bases pour la suite de l'expérimentation relative à la biotolérance des plantes médicinales chez les lapins.

Cependant l'ensemble des données observées avec les paramètres sériques chez les lapins en zone tropicale nécessiterait une autre évaluation sur un échantillonnage statistiquement plus représentatif. Cette évaluation serait un outil indispensable pour des études comparatives avec des valeurs observées sur des lapins élevés hors des zones tropicales d'une part et d'autre part servirait de base de données de référence pour les lapins élevés en zone tropicale.

\section{Références citées}

Adjanohoun E. I. \& Ake A. L., 1979. Contribution au recensement des plantes médicinales de la Côte d'Ivoire. C.R.E.S. Université de Côte d'Ivoire. Centre National de Floristique ; 358 pp.

Boucher S. \& Mouaille L. ,1996. Maladies des lapins - Manuel pratique. Edition France Agricole; $225 \mathrm{pp}$.

Bouyer J., 1997. Méthodes statistiques - Médecine - Biologie. Les éditions INSERM, Paris - France ; $351 \mathrm{pp}$.

Bouyer J., Hemon D., Cordier S., Derriennic F., Stucker I., Stengel B. \& Clavel J., 1995. Epidémiologie - Principes et méthodes quantitatives. Les éditions INSERM, Paris France ; $498 \mathrm{pp}$.

Claude J. R., 1988. Investigation toxicologique pour les nouveaux médicaments. In : Giroud J. P., Matte G. \& Meyniel G., Pharmacologie clinique - Bases thérapeutiques. Expression Scientifique Française.

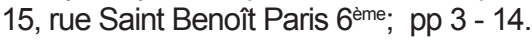

Coulibaly F. A., 2003. Rapport interne non publié. Université de Cocody - Abidjan Côte d'Ivoire. Laboratoire de Pharmacodynamiebiochimique ; 64 pp.

Coulibaly F. A., Djaman J. A., Bahi C. \& GuédéGuina F., 2003. Variation du taux sérique de l'urée et la créatinine chez les lapins traités par Mitagyna ciliata (MYTA) un antipaludique naturel en comparaison avec la chloroquine. Rev Med .Pharm Afr, 18 (2): 31 - 37.

Dagnelie P., 1975. Théorie et méthodes statistiques.2 ${ }^{\text {ème }}$ édition des presses agronomiques de Gembloux, Belgique. 463 pp.

Djédjé H., 2002. Etude prospective pour la réduction et la stabilisation de la glycémie chez le lapin diabétique par DIACODA, des substances de source végétale. Mémoire de D.E.A. Pharmacologie des substances naturelles Université de Cocody- Abidjan, 34 pp.

Eastham R. D., 1978. Abrégé de constantes biologiques. Edition Masson. Paris - France 248 pp. 
Fokouo K. G., 2001. Influence de trois phytomédicaments $\mathrm{HYPO}^{+}$antihypertensif, BGG antidiarrhéique, CX antipaludique sur les concentrations sériques de la créatinine et de l'urée chez les lapins. Mémoire de D.E.A. Pharmacologie des substances naturelles, Université de Cocody - Abidjan, 44 pp.

Kadja B., 1999. Evaluation de quelques paramètres de diagnostique au cours des essais thérapeutiques d'un phytomédicament. Mémoire de D.E.A. de pharmacologie des substances naturelles, Université de Cocody - Abidjan, 31 pp.

Konin K. G., 2002. Evaluation de la concentration sérique de sodium et de potassium chez le lapin traité par deux phytomédicaments anti-infectieux (Thos et MISCA). Mémoire de D.E.A., université de Cocody - Abidjan, 30 pp.
Konrad D. \& Cornélius L. (1972). Tables

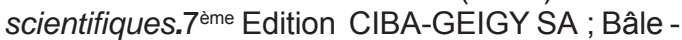
Suisse ; 819 pp.

Laroche M. J. \& Rousselet F., 1990. Le lapin in : Les animaux de laboratoire - Ethiques et bonnes pratiques. Edition : Masson, Paris - France ; pp $219-241$

Lebas F., 2002. Biologie $d u$ lapin. http:// www.cunicultureinfo/Docs indexbiol.htm (accès 20 / 12 / 2005).

Robert J-F., 1980. Constantes biologiques courantes. Les Presses de la SIPE à Rungis (France), 70 pp.

Sibernagl S. \& Despapoulos A., 1992. Atlas de physiologie. Édition Fammarion Médecine Sciences. Paris - France ; 366 pp 